\title{
Can gamma entrainment of the brain rhythms prevent or alleviate Alzheimer's disease?
}

\author{
Tae Kim \\ Department of Biomedical Science and Engineering \\ Gwangju Institute of Science and Technology, Gwangju, Republic of Korea
}

Alzheimer's disease (AD) is the most common cause of dementia, associated with neurobiological markers for progressive structural and functional abnormalities such as synaptic loss, cortical atrophy, and impaired oscillations due to aberrant brain networks. ${ }^{[1]}$ Based on known pathomechanisms, that is, amyloid pathology and tauopathy, there has been a plethora of research to develop an effective pharmacotherapy for AD. ${ }^{[2]}$ Although recently, aducanumab was approved by the Food and Drug Administration (FDA) of the USA, the pharmacologic approach to reduce amyloid-beta $(A \beta)$ for $A D$ is still controversial. ${ }^{[3]}$ In these circumstances, the necessities for non-pharmacologic interventions are being raised. ${ }^{[4]}$

Reduced gamma synchronization in patients with $\mathrm{AD}$ and multiple $\mathrm{AD}$ mouse models led to the hypothesis that aberrant gamma oscillations might be causally related to $A \beta$ pathology. Optogenetically driven neuronal activities at $40 \mathrm{~Hz}$ could result in reduced $\mathrm{A} \beta$ levels in the hippocampus. ${ }^{[5]}$ Furthermore, gamma entrainment by sensory systems, such as visual and auditory, could successfully ameliorate $A \beta$ pathology. ${ }^{[6]}$ The potential mechanism of these remarkable therapeutic effects could be supported by the phenotype change of microglia, a type of immune cell in the central nervous system. There are two polarizations of microglia: M1 is pro-inflammatory and M2 is anti-inflammatory and phagocytic, although the phenotype state is not static, and the microglia switch continuously between the two phenotypes. During the pathogenesis of $\mathrm{AD}$, the progression of dementia is dependent on accumulated $A \beta$ plaques, hyperphosphorylated tau proteins, and activated microglia. ${ }^{[7]}$ Aggregated $A \beta$ acts as a ligand for toll-like receptor 4 (TLR4) on microglia and induces the M1 phenotype of microglia, which secrets pro-inflammatory cytokines. ${ }^{[8]}$ Gamma synchronization enhances phagocytic activity of microglia in 5XFAD mice and increases transcription of the microglial engulfment-associated genes, including Cd68, B2m, Icam1, and Lyz2. ${ }^{[5]}$ By the phagocytic and anti-inflammatory action of $M 2$ microglia, $A \beta$ levels were reduced in animal studies. Moreover, the transcriptomic and phosphoproteomic analysis showed that chronic gamma entrainment also changed neurons per se to be resilient from neurodegeneration by ameliorating synaptic function, increasing neuroprotective factors, and reducing DNA damage in neurons. ${ }^{[0]}$

Given that gamma entrainment has therapeutic potential, what if there is a "center" driving gamma oscillations in the brain? Indeed, the parvalbumin (PV)-containing neurons in the basal forebrain $(\mathrm{BF})$ regulate the cortical gamma oscillations. ${ }^{[10]}$ These neurons project to the cortical interneurons, and they could drive cortical gamma oscillations with a maximal increase at $40 \mathrm{~Hz}$ when stimulated optogenetically. Conversely, the inhibition of these neurons could reduce the auditory steady-state response (ASSR) at $40 \mathrm{~Hz}$ that was transferred to the cortex via $\mathrm{BF}$. Therefore, BF PV neurons can be an potential target to induce strong cortical gamma oscillations in an AD mouse model. However, a recent report showed an 
increased A $\beta 42$ in the prefrontal cortical area and suggested the induction mechanism of cortical gamma oscillations. ${ }^{[11]}$ In fact, fold change of cortical gamma power may be affected sensitively by the length of the individual light pulse (pulse width). ${ }^{[10]}$ Consequently, gamma entrainment may have a potential risk of being harmful and should be carefully designed to be used safely as a therapeutic method.

Gamma entrainment therapy has expandability in terms of entraining modality and target diseases. In addition to the sensory system previously mentioned, various types of neuromodulatory tools can operate as a vehicle for delivering the entraining oscillations. Recently, transcranial alternate current stimulation (tACS) has been suggested as a therapeutic tool. ${ }^{[12]}$ Ultrasound pulsed at gamma frequency can also entrain the brain rhythms noninvasively in $\mathrm{AD}{ }^{[13]}$. A clinical trial was performed with fast gamma magnetic stimulation, although no statistically significant improvement in cognition, functionality, and depression was found. ${ }^{[14]}$ Furthermore, transcutaneous vagus nerve stimulation showed potential benefits for tinnitus by increased gamma band power.

Schizophrenia and autism spectrum disorders can be other potential target diseases for gamma entrainment therapy, given that abnormal gamma oscillations are common in the patients. ${ }^{[15,16]}$ Excitation/inhibition (E/I) imbalance has been suggested as a common pathomechanism of the two diseases. Information processing in the brain is controlled by a functional balance between excitatory and inhibitory networks. The ratio of excitatory versus inhibitory synaptic inputs in the individual cells critically affects the network $\mathrm{E} / \mathrm{I}$ balance and eventually regulates the firing rates of neurons. ${ }^{[1]]}$ Therefore, gamma entrainment may have a therapeutic benefit if it can normalize the impaired E/I balance by driving inhibitory networks.

With the pros and cons of gamma entrainment as a therapy, there are many things to be elucidated before being used clinically. Nevertheless, we may find a positive perspective for translating basic research into clinical applications. 1) Noninvasive approach is possible. As discussed earlier, quite a few approaches are being tested, including sensory stimuli, tACS, ultrasound, and vagus nerve stimulation. Noninvasiveness makes this therapeutic option more feasible and applicable. 2) Side effects are minimally reported yet. Although potential harm could be caused by interfering with the normal mechanism of gamma oscillations, refining the gamma entrainment protocol and selecting an appropriate stimulation modality may minimize the risk. 3) Long-term use may be possible. Neuromodulation for $\mathrm{AD}$ as a preventive measure necessitates long-term use because of chronic disease progression, including AD and other potential applicable diseases. Noninvasiveness and minimal side effects may enable long-term use safely. 4) Physiologic functions are enhanced without external substances. Gamma entrainment may shift microglia from pro-inflammatory to anti-inflammatory polarization.

In summary, gamma entrainment of the brain rhythms has the potential to be a therapeutic methodology for $\mathrm{AD}$ and should be expanded to other neuropsychiatric disorders using various neuromodulation modalities.

\section{Conflict of Interest}

None to declare.

\section{REFERENCES}

1. Knopman DS, Amieva H, Petersen RC, Chételat G, Holtzman DM, Hyman BT, et al. Alzheimer disease. Nat Rev Dis Primers 2021;7:33.

2. Huang LK, Chao SP, Hu CJ. Clinical trials of new drugs for Alzheimer disease. J Biomed Sci 2020;27:18.

3. Liu KY, Howard R. Can we learn lessons from the FDA's approval of aducanumab?. Nat Rev Neurol 2021;17:715-722.

4. Berg-Weger M, Stewart DB. Non-Pharmacologic Interventions for Persons with Dementia. Mo Med 2017;114:116-119.

5. Iaccarino HF, Singer AC, Martorell AJ, Rudenko A, Gao F, Gillingham $\mathrm{TZ}$, et al. Gamma frequency entrainment attenuates amyloid load and modifies microglia. Nature 2016;540:230-235.

6. Martorell AJ, Paulson AL, Suk HJ, Abdurrob F, Drummond GT, Guan W, et al. Multi-sensory Gamma Stimulation Ameliorates Alzheimer's-Associated Pathology and Improves Cognition. Cell 2019;177:256-271.e222.

7. Bolós M, Perea JR, Avila J. Alzheimer's disease as an inflammatory disease. Biomol Concepts 2017;8:37-43.

8. Go M, Kou J, Lim JE, Yang J, Fukuchi KI. Microglial response to LPS increases in wild-type mice during aging but diminishes in an Alzheimer's mouse model: Implication of TLR4 signaling in disease progression. Biochem Biophys Res Commun 2016;479:331-337.

9. Adaikkan C, Middleton SJ, Marco A, Pao PC, Mathys H, Kim DN, et al. Gamma Entrainment Binds Higher-Order Brain Regions and Offers Neuroprotection. Neuron 2019;102:929-943.e928.

10. Kim T, Thankachan S, McKenna JT, McNally JM, Yang C, Choi JH, et al. Cortically projecting basal forebrain parvalbumin neurons regulate cortical gamma band oscillations. Proc Natl Acad Sci U S A 2015;112:3535-3540.

11. Wilson CA, Fouda S, Sakata S. Effects of optogenetic stimulation of basal forebrain parvalbumin neurons on Alzheimer's disease pathology. Sci Rep 2020;10:15456.

12. Strüber D, Herrmann CS. Modulation of gamma oscillations as a possible therapeutic tool for neuropsychiatric diseases: A review and perspective. Int J Psychophysiol 2020;152:15-25.

13. Park M, Hoang GM, Nguyen T, Lee E, Jung HJ, Choe Y, Lee MH, Hwang JY, Kim JG, Kim T. Effects of transcranial ultrasound stimulation pulsed at $40 \mathrm{~Hz}$ on $\mathrm{A} \beta$ plaques and brain rhythms in $5 \times \mathrm{FAD}$ mice. Transl Neurodegener. 2021;10:48.

14. Mimenza-Alvarado AJ, Aguilar-Navarro SG, Martinez-Carrillo FM, Ríos-Ponce AE, Villafuerte G. Use of Fast Gamma Magnetic Stimulation Over the Left Prefrontal Dorsolateral Cortex for the Treatment of MCI and Mild Alzheimer's Disease: A Double-Blind, Randomized, ShamControlled, Pilot Study. Front Neurol 2021;12:729-872. 
Kim: Can gamma entrainment of the brain rhythms prevent or alleviate Alzheimer's disease?

15. McNally JM, McCarley RW. Gamma band oscillations: a key to understanding schizophrenia symptoms and neural circuit abnormalities. Curr Opin Psychiatry 2016;29:202-210.

16. Rojas DC, Wilson LB. $\gamma$-band abnormalities as markers of autism spectrum disorders. Biomark Med 2014;8:353-368.

17. Gao R, Penzes P. Common mechanisms of excitatory and inhibitory imbalance in schizophrenia and autism spectrum disorders. Curr Mol Med 2015;15:146-167.

How to cite this article: Kim T. Can gamma entrainment of the brain rhythms prevent or alleviate Alzheimer's disease? J Transl Intern Med 2021; 9: 231-3. 